\title{
SV2A PET imaging is a non-invasive marker for the detection of spinal damages in experimental models of spinal cord injury
}

\author{
Daniele Bertoglio ${ }^{1,2}$, Nicolas Halloin ${ }^{3}$, Stef De Lombaerde ${ }^{1,4}$, Aleksandar Jankovski ${ }^{5,6}$, \\ Jeroen Verhaeghe ${ }^{1}$, Charles Nicaise ${ }^{3, *}$ and Steven Staelens ${ }^{1,2, *}$ \\ *last shared author
}

1. Molecular Imaging Center Antwerp (MICA), University of Antwerp, Belgium

2. $\mu$ NEURO Research Centre of Excellence, University of Antwerp, Antwerp, Belgium

3. URPhyM - NARILIS, University of Namur, Belgium

4. Department of Nuclear Medicine, Antwerp University Hospital, Belgium

5. Institute of NeuroScience, NEUR division, Université Catholique de Louvain, Belgium

6. Department of Neurosurgery, CHU UCL Namur, Yvoir, Belgium

\section{Corresponding author:}

Prof. Steven Staelens

MICA, University of Antwerp, Belgium

Tel. +32032652820; Email: steven.staelens@uantwerpen.be

\section{First author:}

Daniele Bertoglio, $\mathrm{PhD}$

MICA, University of Antwerp, Belgium

Tel. +32032652816; Email: daniele.bertoglio@uantwerpen.be

Orcid: https://orcid.org/0000-0003-4205-5432

Word count: 5062 words

Short running title: SV2A as a biomarker for $\mathrm{SCl}$

Immediate Open Access: Creative Commons Attribution 4.0 International License (CC BY) allows users to share and adapt with attribution, excluding materials credited to previous publications.

License: https://creativecommons.org/licenses/by/4.0/. Details: https://jnm.snmjournals.org/page/permissions.

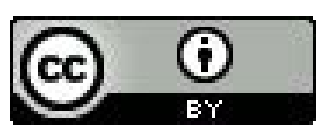




\section{ABSTRACT}

Traumatic spinal cord injury $(\mathrm{SCl})$ is a neurological condition characterized by long-term motor and sensory neurological deficits as a consequence of an external physical impact damaging the spinal cord. Anatomical magnetic resonance imaging (MRI) is considered the gold-standard diagnostic tool to obtain structural information for the prognosis of acute $\mathrm{SCl}$, however, it lacks functional objective information to assess $\mathrm{SCl}$ progression and recovery. In this study, we explored the use of synaptic vesicle glycoprotein 2A (SV2A) positron emission tomography (PET) imaging to detect spinal cord lesions non-invasively following SCl.

METHODS: Mice $(n=7)$ and rats ( $n=8)$ subjected to unilateral moderate cervical (C5) contusion were euthanized 1-week post-SCl for histological and autoradiographic $\left({ }^{3} \mathrm{H}\right.$ UCB-J) investigation of SV2A levels. Longitudinal ${ }^{11} \mathrm{C}-U C B-J$ PET/computed tomography (CT) imaging was performed in sham $(n=7)$ and $\mathrm{SCI}$ rats $(n=8) 1$ week and 6 weeks post-SCI. Animals also underwent an ${ }^{18} \mathrm{~F}$-FDG PET scan during the latter time point. Post-mortem tissue SV2A analysis to corroborate in vivo PET findings was performed 6 weeks post-SCl.

RESULTS: A significant SV2A loss (ranging from $-70.3 \%$ to $-87.3 \% ; P<0.0001$ ) was measured at the epicenter of the impact in vitro in both mouse and rat contusion $\mathrm{SCl}$ models. Longitudinal ${ }^{11} \mathrm{C}-\mathrm{UCB}-\mathrm{J}$ PET imaging detected SV2A loss in SCl rats ($49.0 \pm 8.1 \%$ at 1 week and $-52.0 \pm 12.9 \%$ at 6 weeks post-SCI), with no change observed in sham rats. In contrast, ${ }^{18} \mathrm{~F}$-FDG PET imaging measured only subtle hypometabolism (-17.6 $\pm 14.7 \%)$. Finally, post-mortem ${ }^{3} \mathrm{H}-\mathrm{UCB}-\mathrm{J}$ autoradiography correlated with the in vivo SV2A PET findings $(\mathrm{r}=0.92, P<0.0001)$.

CONCLUSION: ${ }^{11} \mathrm{C}-U C B-J$ PET/CT imaging is a non-invasive marker for SV2A loss following SCl. Collectively, these findings indicate SV2A PET may provide an objective measure of $\mathrm{SCl}$, and thus a valuable tool to evaluate novel therapeutics. Clinical assessment of SCl with SV2A PET imaging is highly recommended.

Keywords: SV2A; ${ }^{11}$ C-UCB-J; biomarker, contusion SCI; animal model 


\section{INTRODUCTION}

Spinal cord injury $(\mathrm{SCl})$ is a devastating condition characterized by long-term motor and sensory neurological deficits as well as a strong impact on the cognitive and social life of patients (1). Traumatic $\mathrm{SCl}$ is the consequence of an external physical impact damaging the spinal cord, with the neurological outcomes being related to the extent of the primary lesion $(1,2)$.

Currently, magnetic resonance imaging (MRI) is considered the gold-standard diagnostic tool to obtain structural information on spinal cord lesion and neighboring tissues during the acute phase of $\mathrm{SCl}(3-5)$. While routine 1.5T MRI offers a clear view of the cord edema, hemorrhage, or syrinx formation, yet it has been shown to inconsistently correlate with the neurological findings (6-8). Since MRI-based prognostication, treatment, and rehabilitation planning of spinally injured patients remains a daily clinical challenge (9), there has been an increasing interest in developing objective imaging modalities to assess $\mathrm{SCl}$ progression and recovery for the non-invasive assessment of emerging therapeutic approaches (4-6).

Positron emission tomography (PET) imaging of glucose metabolism using ${ }^{18} \mathrm{~F}-\mathrm{FDG}$ may represent an imaging modality to monitor metabolic activation and functional neuronal viability at the trauma epicenter. ${ }^{18} \mathrm{~F}$-FDG PET imaging has been performed in both animal models and $\mathrm{SCl}$ patients, demonstrating abnormal metabolic activity in animals during the acute phase of experimental $\mathrm{SCl}$ or in patients during the active phases of compressive myelopathies (10-15). Although useful, the significance of the ${ }^{18} \mathrm{~F}-\mathrm{FDG}$ signal remains flawed by its unspecific nature, mixing neuronal metabolism and glial activation (16), and by the lack of correlation with neurological functions (17).

Recently, novel radioligands targeting the synaptic vesicle glycoprotein 2A (SV2A), a presynaptic protein regulating neurotransmitter release (18) have been developed based on the antiepileptic drug levetiracetam (19). These ligands, including the selective and high-affinity radioligand ${ }^{11} \mathrm{C}-\mathrm{UCB}-\mathrm{J}$, have emerged as powerful tools to quantify synaptic density in vivo in a non-invasive manner $(20,21)$. SV2A PET imaging has already proven a useful tool for assessing 
synaptic density in various neurodegenerative and neuropsychiatric disorders at clinical and preclinical stages (22-24).

Although SV2A PET imaging has been mainly applied to the brain, SV2A is also present in the grey matter of the spinal cord (25). Furthermore, we recently showed ${ }^{11} \mathrm{C}-\mathrm{UCB}-\mathrm{J}$ PET imaging can be used to quantify SV2A density non-invasively in the rodent spinal cord (23). We, therefore, postulated that SV2A PET imaging could serve as a biomarker of synaptic loss following spinal cord injury (SCI). In this study, we explored SV2A levels in experimental models of cervical contusion $(26,27)$, investigated the potential of non-invasive ${ }^{11} \mathrm{C}-\mathrm{UCB}-\mathrm{J}$ PET imaging to detect spinal damage in rats following $\mathrm{SCI}$, and compared ${ }^{11} \mathrm{C}-\mathrm{UCB}-\mathrm{J}$ and ${ }^{18} \mathrm{~F}$-FDG PET imaging to verify whether SV2A PET could provide enhanced specificity and sensitivity in detecting spinal injury. 


\section{MATERIALS AND METHODS}

\section{Animals and Experimental Design}

All experiments were conducted in compliance with the European Communities Council Directive (2010/63/EU), and granted authorizations from the local animal ethic committees (University of Namur, UN19-339 and University of Antwerp, ECD2019-49). Experiments outcomes were carried out at 1-week post-SCI (sub-acute phase) and 6 weeks post-SCI (chronic phase), as outlined in Supplemental Figure 1. First, we investigated in vitro both mouse and rat models to assess whether SV2A levels were affected differentially between species. For the longitudinal in vivo study, the rat model was preferred given its favourable body and spinal cord size compared to mice. Nonetheless, investigation in mice is relevant given the abundance of genetic mouse models with relevant spinal cord diseases $(23,28)$.

Adult male C57BL/6J mice ( $n=7 ; 20-25 \mathrm{~g}$ ) (internal breeding from the University of Namur) and female Sprague-Dawley rats ( $n=8 ; 225-275 \mathrm{~g})$ (Charles River Laboratories, France) underwent SCl and were euthanatized 1-week post-SCI for histological and autoradiographic assessment of SV2A. Additional fifteen female Sprague-Dawley rats (225-275 g) were subjected either to $\mathrm{SCI}(n=8)$ or laminectomy only (sham, $n=7)$ and were included in the longitudinal PET study and euthanatized at 6 weeks post-SCI. Animals were group-housed under a 12h light/dark cycle in a temperature- and humidity-controlled environment with food and water ad libitum.

\section{Contusion Spinal Cord Injury}

Animals underwent a cervical C5 unilateral right-sided contusion as previously described for mice (26) and rats (27). Animals were anesthetized using a mix of ketamine $100 \mathrm{mg} / \mathrm{kg}$ (Ketamine 1000 Ceva, Belgium) and xylazine 5mg/kg (XYL-M 2\% VMD, Arendonk, Belgium). Briefly, dorsal skin and underlying muscles were cut to get access to the spine between the spinous processes $\mathrm{C} 2$ and T1. The paravertebral muscles overlying C3-C5 were carefully removed. Animals were then subjected to laminectomy at the C5 vertebra before receiving a 
computer-controlled impact of 51.3 \pm 1.7 kDynes for mice and 407 \pm 6.1 kDynes for rats (dwell time of $0 \mathrm{~s}$ ) using IH Spinal Cord Impactor (Precision Systems and Instrumentation, LLC, Sarasota, USA). The full procedure included unilateral laminectomy on the right side, clamping of the spinous processes of C2 and T2 using toothed Adson forceps to stabilize the whole spinal column, raising of the impactor tip (1.0 mm diameter impactor for mice and $1.5 \mathrm{~mm}$ for rats) $2.5 \mathrm{~mm}$ above the dura, and contusion of the right hemicord (bathed in $0.9 \%$ sterile saline for rats). Muscles were sutured in layers using silk 2.0 (mice) or 4.0 (rats) and the skin closed using surgical clippers. Animals received buprenorphine (s.c.; Vetergesic Ceva, Belgium) $0.05 \mathrm{mg} / \mathrm{kg}$ for analgesia after surgery, and again 12 and $24 \mathrm{~h}$ post-surgery. Saline was subcutaneously administered for rehydration immediately after surgery and 24 hours later. One $\mathrm{SCl}$ rat did not recover from the surgical procedure and was euthanized. Sham animals underwent the full laminectomy procedure without contusion.

\section{Radiosynthesis}

${ }^{11} \mathrm{C}-U C B-J$ synthesis was performed on an automated synthesis module (Carbosynthon I, Comecer, The Netherlands) as previously described $(20,23)$ with an average molar activity of 52.9 $\pm 12.4 \mathrm{GBq} / \mu \mathrm{mol}$. Radiochemical purity was greater than $99 \%$ for both ${ }^{11} \mathrm{C}-\mathrm{UCB}-\mathrm{J}$ and ${ }^{18} \mathrm{~F}$ FDG. ${ }^{3} \mathrm{H}-U C B-J$ (Novandi Chemistry AB, Sweden) was synthetized with molar radioactivity of 2997 $\mathrm{MBq} / \mu \mathrm{mol}$ and radiochemical purity $>99 \%$.

\section{MicroPET Imaging}

MicroPET/Computed tomography (CT) imaging was performed on two Siemens Inveon PET/CT scanners (Siemens Preclinical Solution, USA). Animal preparation was performed as previously described (29). For the ${ }^{18}$ F-FDG PET scan, animals were fasted the evening before with a fasting period of $17.7 \pm 1.6 \mathrm{~h}$. Glucose concentration $(81.2 \pm 15.1 \mathrm{mg} / \mathrm{dl})$ was measured from blood samples obtained from the tail vein of the rats before radioligand injection using glucose 
strips in duplicate (One Touch Ultra 2, Lifescan). Rats were anesthetized using isoflurane (Forene, Belgium) in medical oxygen (induction 5\%, maintenance $2-2.5 \%$ ). The respiration rate and body temperature were monitored and maintained constant during the entire scan. ${ }^{11} \mathrm{C}-\mathrm{UCB}-\mathrm{J}$ PET images were acquired dynamically (50 min scan), whereas static (20 min) PET scans were performed for glucose metabolism $\left({ }^{18} \mathrm{~F}-\mathrm{FDG}\right)$ following $30 \mathrm{~min}$ of awake radioligand uptake $(29,30)$. Animals were injected into a catheterized tail vein over a 30 -second interval $(1 \mathrm{ml} / \mathrm{min})$ using an automated pump (Pump 11 Elite, Harvard Apparatus, USA) with a bolus of ${ }^{11} \mathrm{C}-\mathrm{UCB}-\mathrm{J}$ $(6.7 \pm 4.0 \mathrm{MBq})$ or ${ }^{18} \mathrm{~F}-\mathrm{FDG}(25.1 \pm 2.0 \mathrm{MBq})$. Data were acquired in list-mode format. Following the microPET scan, a 10 min $80 \mathrm{kV} / 500 \mu \mathrm{A}$ CT scan was performed for co-registration and attenuation correction.

\section{Image Processing and Analysis}

${ }^{11} \mathrm{C}-U C B-J$ data were reconstructed into 31 frames of increasing length $(12 \times 10 \mathrm{~s}, 3 \times 20 \mathrm{~s}$, $3 \times 30 \mathrm{~s}, 3 \times 60 \mathrm{~s}, 3 \times 150 \mathrm{~s}$, and $7 \times 300 \mathrm{~s})$, while a static reconstruction was applied to ${ }^{18} \mathrm{~F}-\mathrm{FDG}$ data using 8 iterations and 16 subsets of the 3D ordered subset expectation maximization algorithm with spatially variant modeling (31) with normalization, dead time, and CT-based attenuation corrections. Images were reconstructed on a $128 \times 128 \times 159$ grid with $0.776 \times 0.776 \times 0.796 \mathrm{~mm}^{3}$ voxels. PET/CT images were processed and analyzed using PMOD 3.6 software (Pmod Technologies, Zurich, Switzerland).

To align all brains to a common space and use the same volume-of-interest (VOI) to extract the whole-brain activity, brain PET images were rigid matched to the Schiffer rat ${ }^{18} \mathrm{~F}$-FDG template (32) or the ${ }^{11}$ C-UCB-J PET template (generated in the same space of the Schiffer one). Cervical spinal cord VOls were manually delineated using the individual co-registered PET/CT images from C3 to C7 with C5 sub-divided into right and left segments. Since we anticipated a potential partial volume effect on the contralateral C5 segment, comparisons were made against the C3 section. The VOIs were kept of the same volume across subjects $\left(0.016 \mathrm{~cm}^{3}\right.$ each $\mathrm{C} 3-\mathrm{C} 7$ level and 0.008 
$\mathrm{cm}^{3}$ for left and right C5). Across animals, VOls were manually reoriented to follow the profile of the spine. In Supplemental Figure 2 the CT image of the spine of a representative sham rat with laminectomy is shown.

For ${ }^{11} \mathrm{C}-U C B-J$ analysis, the time interval $25-45$ min p.i. was selected to determine radioligand uptake measured using standardized uptake value (SUV), calculated as brain activity multiplied by body weight and divided by the injected dose. No difference in cerebral uptake was observed for either ${ }^{11} \mathrm{C}$-UCB-J (SUV) (Supplemental Figure 3) or ${ }^{18} \mathrm{~F}-\mathrm{FDG}$ (SUV glc, SUV corrected for glucose levels) (Supplemental Figure 4), therefore spinal uptake was measured as the ratio of regional spinal uptake over the whole-brain uptake.

\section{Histological Analysis}

One- or 6-weeks post-injury, animals were deeply anesthetized with sodium pentobarbital (50 mg/kg, intraperitoneal) and transcardially perfused with ice-cold phosphate buffer saline (PBS, $\mathrm{pH}$ 7.4). Spinal cords were dissected and a cervical segment containing the contusion injury was snap-frozen in 2 -metylbuthane at $-35^{\circ} \mathrm{C}$ for $2 \mathrm{~min}$ and preserved at $-80^{\circ} \mathrm{C}$ until use. Coronal sections (30 $\mu \mathrm{m}$ of thickness) were collected on Superfrost plus slides (Thermo Fischer Scientific, USA), using a cryostat (Leica, Germany), and distributed into a series of 12 slices so that each slide covered rostral, trauma, and caudal sections.

SV2A immunostaining was performed as previously described (23) using the primary antibody anti-SV2A (rabbit IgG; 1:400; \#66724, Cell signalling technologies), followed by the secondary fluorescent antibody (donkey anti-rabbit; 1:100; Alexa Fluor 488, Jackson ImmunoResearch). Images were acquired at 10X magnification for quantification with a fluorescence microscope (Olympus, Japan) using CellSens software. Image analysis was performed using Fiji (v2.1.0) ImageJ software (National Institute of Health, USA). SV2A signal intensity was measured in regions-of-interest (right and left grey matter spinal cord) manually delineated on each section. Hematoxylin and eosin (H\&E) staining was performed on adjacent 
slides to provide morphological visualization of tissue.

\section{Autoradiography}

In vitro ${ }^{3} \mathrm{H}-\mathrm{UCB}-\mathrm{J}$ (Novandi Chemistry $\mathrm{AB}$, Sweden) autoradiography was performed as previously reported (23). Mouse and rat SV2A selectivity in the spinal cord was validated using a blocking solution ( $1 \mathrm{nM}$ of ${ }^{3} \mathrm{H}-\mathrm{UCB}-\mathrm{J}+1 \mathrm{mM}$ of levetiracetam in binding buffer) (Supplemental Figure 5).

Regional quantification was performed using Fiji (v2.1.0) ImageJ software. Specific binding of ${ }^{3} \mathrm{H}-\mathrm{UCB}-\mathrm{J}$ was measured by converting the mean grey values into radioactivity density $(\mathrm{Bq} / \mathrm{mg})$ calculated using commercial tritium standards (American Radiolabeled Chemicals). Next, using ${ }^{3} \mathrm{H}-U \mathrm{CB}-\mathrm{J}$ molar activity on the experimental day, radioactivity density was converted into binding density (pmol/mg) for each region.

\section{Statistical Analysis}

In vivo spinal cord PET data, as well as post-mortem analyses, were investigated using a two-way ANOVA (experimental group and region as variables). Spinal PET signal decrease compared to C3 and cerebral binding were assessed using the Mann-Whitney test. Autoradiographic blocking experiments were analyzed using paired T-tests. Correlations between in vivo and post-mortem SV2A signal were evaluated using a Spearman $r$ test. $P$ values were corrected for multiple comparisons using Šídák's multiple comparisons test. Statistical analyses were performed with GraphPad Prism (v9.1) statistical software. Data are represented as mean \pm standard deviation (SD). All tests were two-tailed and statistical significance was set at $P<0.05$. 


\section{RESULTS}

\section{SV2A Levels are Decreased Sub-Acutely in Experimental Models of Contusion SCI}

To determine whether SV2A levels are changed following a spinal injury, we performed SV2A histological and autoradiographic assessment in two experimental models of contusion SCI during the sub-acute phase (Figure $1 \mathrm{~A}$ and Supplemental Figure $6 \mathrm{~A}$ ). In both mouse and rat spinal cord, a significant SV2A loss at the epicenter of impact, but not in rostral sections (C3-C4 level), was observed with SV2A immunostaining (contralateral effect: mouse: $F_{(1,24)}=140.4, P<0.0001$; rat: $\left.F_{(1,28)}=20.2, P<0.0001\right)$, which corresponded to around $80 \%$ decline compared to rostral sections (mouse $=-81.2 \pm 8.6 \%$, post-hoc: $P<0.0001$; rat $=-78.8 \pm 21.1 \%$, post $-h o c: P<0.0001$ ) (Figure 1B and Supplemental Figure 6B). Analogously, ${ }^{3} \mathrm{H}-\mathrm{UCB}-\mathrm{J}$ binding was significantly affected at the epicenter of the contusion compared to contralateral (contralateral effect: mouse: $F_{(1,24)}=42.3, P<0.0001$; rat: $\left.F_{(1,28)}=24.4, P<0.0001\right)$, with a clear decline in relation to rostral sections (mouse $=-70.3 \pm 11.5 \%$, post-hoc: $P<0.0001 ;$ rat $=-87.3 \pm 10.9 \%$, post $-h o c: P<0.0001$ ) (Figure 1C and Supplemental Figure 6C). Notably, the SV2A loss was not due to loss of spinal tissue as depicted by the H\&E staining.

\section{${ }^{11}$ C-UCB-J PET Detects In Vivo SV2A Loss Following SCI}

Given the wider diameter of the spinal cord in the rat, the rat model was chosen to investigate ${ }^{11} \mathrm{C}$-UCB-J PET imaging following SCI. Figure 2 displays representative ${ }^{11} \mathrm{C}-\mathrm{UCB}-\mathrm{J}$ uptake images of $\mathrm{SCl}$ rat compared to sham control 1-week post-injury. Spinal cord ${ }^{11} \mathrm{C}-\mathrm{UCB}-\mathrm{J}$ uptake at the contusion site (right C5) was significantly changed compared to sham animals (group effect: $F_{(1,54)}=100.2, P<0.0001$ ) (Figure 3A). ${ }^{11} \mathrm{C}$-UCB-J uptake was also significantly reduced in surrounding areas (C4, left $\mathrm{C} 5, \mathrm{C} 6)$. Accordingly, SCl animals displayed ${ }^{11} \mathrm{C}-\mathrm{UCB}-\mathrm{J}$ binding decline in right C5 compared to C3 $(-49.0 \pm 8.1 \%)$, which significantly differed from sham rats $(P=0.0043)$ (Figure 3B). 


\section{${ }^{11}$ C-UCB-J PET Outperforms ${ }^{18}$ F-FDG PET for Detection of chronic SCI}

Similarly to 1 -week post-injury, reduction of ${ }^{11} \mathrm{C}-U \mathrm{CB}-\mathrm{J}$ uptake around the contusion site (right $\mathrm{C5}$ ) in $\mathrm{SCl}$ rats was significant and sustained at 6-weeks post-injury compared to sham animals (group effect: $F_{(1,60)}=190.4, P<0.0001$ ) (Figure $4 \mathrm{~A}$ ). Hence, SCl animals displayed ${ }^{11} \mathrm{C}$ UCB-J binding decline in right C5 compared to C3 (-52.0 $\pm 12.9 \%)$, which significantly differed from sham rats $(P=0.0025)$ (Figure $4 \mathrm{~B})$. Interestingly, ${ }^{11} \mathrm{C}-\mathrm{UCB}-\mathrm{J}$ uptake at chronic trauma in $\mathrm{SCI}$ rats was significantly affected until $\mathrm{C} 7(P<0.01)$, unlike at 1 -week post-SCI $(P>0.05)$ suggesting that the local contusion might induce SV2A loss caudal to the lesion epicenter.

Six weeks post-SCI, animals underwent ${ }^{11} \mathrm{C}-\mathrm{UCB}-\mathrm{J}$ and ${ }^{18} \mathrm{~F}-\mathrm{FDG}$ PET scans to assess whether SV2A PET could provide enhanced sensitivity in detecting spinal injury. ${ }^{18} \mathrm{~F}$-FDG PET could detect hypometabolism at the contusion epicenter (right C5) compared to sham animals (group effect: $F_{(1,60)}=70.9, P<0.0001$ ) (Figure $5 \mathrm{~A}$ ) as well as hypometabolism in surrounding tissue (left C5, C6). However, the difference compared to C3 was subtle $(-17.6 \pm 14.7 \%)$, with no significant change from sham rats $(P=0.106)$ (Figure 5B).

\section{Post-Mortem Analysis Corroborates ${ }^{11}$ C-UCB-J PET Findings}

Similar to the analyses at the sub-acute phase, a significant SV2A loss at the epicenter, but not in rostral (C3-C4) sections, was observed at chronic phase with SV2A immunostaining (group effect: $\left.F_{(1,22)}=62.0, P<0.0001\right)$, corresponding to $86 \pm 17.4 \%$ decline compared to rostral sections (Figure 6A), showing strong consistency with the in vivo ${ }^{11} \mathrm{C}-\mathrm{UCB}-\mathrm{J}$ PET measurements $(\mathrm{r}=0.86, P=0.0013$; Figure 6B).

${ }^{3} \mathrm{H}-U \mathrm{CB}-\mathrm{J}$ binding was significantly affected as well (group effect: $F_{(1,22)}=31.2, P<0.0001$ ), with a $82.1 \pm 22.8 \%$ decline compared to rostral sections (Figure $6 \mathrm{C}$ ). ${ }^{3} \mathrm{H}-\mathrm{UCB}-\mathrm{J}$ autoradiography reflected the in vivo ${ }^{11} \mathrm{C}-U C B-J$ PET quantification ( $r=0.92, P<0.0001$; Figure 6D). 


\section{DISCUSSION}

This study showed SV2A is decreased in mouse and rat experimental models of SCI contusion and that synaptic loss following $\mathrm{SCl}$ can be measured non-invasively. Also, SV2A decrease is sustained over time and ${ }^{11} \mathrm{C}-U \mathrm{UCB}-\mathrm{J}$ PET imaging provides a three-fold enhanced sensitivity compared to ${ }^{18} \mathrm{~F}-\mathrm{FDG}$ PET imaging in assessing spinal damage. Collectively, these findings suggest SV2A PET imaging could serve as a robust biomarker capable of detecting subtle synaptic loss following SCI.

Validation by histological and autoradiographic findings confirmed SV2A to be restricted to grey matter (25) and that ${ }^{3} \mathrm{H}-\mathrm{UCB}-\mathrm{J}$ binding was blocked by levetiracetam (19). Both experimental models of SCI displayed a comparable unilateral SV2A loss and, most importantly, SV2A decline was not due to a mere tissue loss caused by the initial trauma.

For in vivo PET quantification, we focused on the comparison to the rostral C3 level which is morphologically unaffected to avoid partial volume effect likely present in the uninjured C5 hemicord. We measured a sustained reduction in ${ }^{11} \mathrm{C}-\mathrm{UCB}-\mathrm{J}$ binding at the SCI epicenter over time, suggesting little-to-none regeneration of synaptic connections and sprouting of spared axons. Notably, a decrease in SV2A was observed in SCI rats over time at the caudal level (C6$\mathrm{C7}$ ), a possible result of the deafferentation of the grey matter and consequent caudal synaptic loss, overall reflecting an extension of the secondary lesion, which comprises many processes including apoptosis, demyelination of surviving axons, axonal die-back, and building of a glial scar around the injury site $(1,33,34)$.

Previous studies have reported only transient changes in ${ }^{18} \mathrm{~F}-\mathrm{FDG}$ uptake in rat and canine models $(13,14)$. Thus, one of the objectives of the current study was to compare the performance of ${ }^{11} \mathrm{C}$-UCB-J to ${ }^{18} \mathrm{~F}-\mathrm{FDG}$ in detecting spinal cord damages. Despite the extensive neurodegeneration occurring at trauma site (1), the observed reduction in glucose uptake was only a fraction (-17.6\%) compared to the SV2A loss (-52.0\%). This is likely related to the enhanced

gliosis driven by microglia/macrophage and astrocyte activation related to primary and secondary 
injuries. Since glucose uptake is the result of neuronal and glial metabolism (16), the gliosis occurring at the trauma site can largely mask the reduced uptake associated with neurodegeneration. As such, understanding the biological process driving the ${ }^{18} \mathrm{~F}$-FDG uptake is very challenging, especially for the assessment of therapeutics, due to the plethora of processes occurring at the injury site $(1,34)$. On the contrary, the precise nature of SV2A as the molecular target of ${ }^{11} \mathrm{C}$-UCB-J offers a specific measure of synaptic density without being affected by secondary processes. Thus, SV2A PET imaging might provide a powerful tool for the assessment of synaptic loss in traumatic, neurodegenerative, inflammatory, and vascular spinal cord pathologies, as well as for synaptic loss, regeneration (24) and axonal connectivity following gene and cell therapy approaches (35). Future studies investigating the association between spinal SV2A density and degree of motor impairment will be needed to understand whether SV2A loss might represent a biomarker with functional relevance.

Despite being the gold-standard diagnostic tool to obtain structural information on spinal cord lesions during the acute phase of $\mathrm{SCI}$ (4), functional assessment based on MRI remains elusive resulting in inconsistent correlates with the neurological findings (6-8). Although novel approaches such as diffusion tensor imaging and myelin water transfer can provide an added value in terms of axon and myelin integrity (4), their readouts are likely to be affected by evolving edema, hemorrhage, or syrinx formation. In this view, SV2A PET imaging can offer an attractive complementary alternative to measure objectively synaptic integrity at the lesion site in preclinical and clinical settings. Although we describe here for the first time spinal SV2A changes, SV2A PET imaging has already been performed in several clinical studies of neurological disorders (22). With a more favorable resolution ratio (spinal cord/scanner resolution) available for human PET imaging, we foresee no limitation for clinical application as supported by a preliminary report in healthy subjects (36). The high sensitivity of SV2A as compared to ${ }^{18}$ F-FDG PET and the added value of ${ }^{18} \mathrm{~F}$-FDG PET as compared to MRI (15) suggest that SV2A PET may be of particular 
interest in the diagnosis and prognosis of cervical spondylotic myelopathy which is a common pathology in the elderly (37).

\section{CONCLUSION}

Using ${ }^{11} \mathrm{C}-U C B-J$ PET/CT imaging, we successfully detected SV2A loss in the contusion $\mathrm{SCl}$ rat model. The presented findings indicate synaptic loss following $\mathrm{SCl}$ can be quantified noninvasively, SV2A decrease is sustained over time, and ${ }^{11} \mathrm{C}-\mathrm{UCB}-\mathrm{J}$ PET imaging provides enhanced sensitivity compared to ${ }^{18}$ F-FDG PET imaging for assessment of spinal damage. Collectively, these findings indicate SV2A PET may provide an objective measure of SCI, and thus a valuable tool to evaluate novel therapeutics. Clinical assessment of SCI with SV2A PET imaging is highly recommended. 


\section{DISCLOSURE}

The authors have no conflicts of interest.

\section{ACKNOWLEDGEMENTS}

DB is supported by the Research Foundation Flanders (FWO, 1229721N). Antwerp University also founded the work through a partial assistant professor position for JV and a full professor position for SS. The authors thank Philippe Joye, Romy Raeymakers, and Annemie Van Eetveldt of MICA for their valuable technical assistance. Part of the graphical abstract and supplemental figure 1 were generated using Biorender.

\section{KEY POINTS}

\section{Question:}

Does SV2A represent a biomarker for spinal cord injury?

\section{Pertinent findings:}

SV2A levels are significantly reduced in animal models of contusion spinal cord injury. Longitudinal ${ }^{11} \mathrm{C}-$-UCB-J PET imaging showed that SV2A loss can be quantified non-invasively, is sustained over time, and outperforms ${ }^{18}$ F-FDG PET imaging.

\section{Implication for patient care:}

SV2A PET imaging offers a novel non-invasive method to quantify $\mathrm{SCl}$, which may provide a promising diagnostic tool for the assessment of functional recovery following SCI therapeutics. 


\section{REFERENCES}

1. Ahuja CS, Wilson JR, Nori S, et al. Traumatic spinal cord injury. Nat Rev Dis Primers. 2017;3:17018.

2. Wilson JR, Hashimoto RE, Dettori JR, Fehlings MG. Spinal cord injury and quality of life: a systematic review of outcome measures. Evid Based Spine Care J. 2011;2:37-44.

3. Freund $\mathrm{P}$, Curt A, Friston K, Thompson A. Tracking changes following spinal cord injury: insights from neuroimaging. Neuroscientist. 2013;19:116-128.

4. Freund P, Seif M, Weiskopf N, et al. MRI in traumatic spinal cord injury: from clinical assessment to neuroimaging biomarkers. Lancet Neurol. 2019;18:1123-1135.

5. Lammertse D, Dungan D, Dreisbach J, et al. Neuroimaging in traumatic spinal cord injury: an evidence-based review for clinical practice and research. J Spinal Cord Med. 2007;30:205-214.

6. Ellingson BM, Salamon N, Holly LT. Imaging techniques in spinal cord injury. World Neurosurg. 2014;82:1351-1358.

7. Chay W, Kirshblum S. Predicting outcomes after spinal cord injury. Phys Med Rehabil Clin N Am. 2020;31:331-343.

8. Sharif S, Jazaib Ali MY. Outcome prediction in spinal cord injury: myth or reality. World Neurosurg. 2020;140:574-590.

9. Talbott JF, Huie JR, Ferguson AR, Bresnahan JC, Beattie MS, Dhall SS. MR Imaging for Assessing Injury Severity and Prognosis in Acute Traumatic Spinal Cord Injury. Radiol Clin North Am. 2019;57:319-339.

10. Flanagan EP, Hunt $\mathrm{CH}$, Lowe $\mathrm{V}$, et al. [(18)F]-fluorodeoxyglucose-positron emission tomography in patients with active myelopathy. Mayo Clin Proc. 2013;88:1204-1212.

11. Floeth FW, Stoffels G, Herdmann J, et al. Prognostic value of 18F-FDG PET in monosegmental stenosis and myelopathy of the cervical spinal cord. J Nucl Med. 2011;52:13851391.

12. Nandoe Tewarie RD, Yu J, Seidel J, et al. Positron emission tomography for serial imaging of the contused adult rat spinal cord. Mol Imaging. 2010;9:108-116.

13. von Leden RE, Selwyn RG, Jaiswal S, Wilson CM, Khayrullina G, Byrnes KR. (18)F-FDGPET imaging of rat spinal cord demonstrates altered glucose uptake acutely after contusion injury. Neurosci Lett. 2016;621:126-132.

14. Zhang L, López-Picón FR, Jia Y, et al. Longitudinal [(18)F]FDG and [(13)N]NH(3) $\mathrm{PET} / \mathrm{CT}$ imaging of brain and spinal cord in a canine hemisection spinal cord injury model. Neuroimage Clin. 2021;31:102692. 
15. Eicker SO, Langen KJ, Galldiks N, et al. Clinical value of 2-deoxy-[18F]fluoro-D-glucose positron emission tomography in patients with cervical spondylotic myelopathy. Neurosurg Focus. 2013;35:E2.

16. Xiang $\mathrm{X}$, Wind $\mathrm{K}$, Wiedemann $\mathrm{T}$, et al. Microglial activation states drive glucose uptake and FDG-PET alterations in neurodegenerative diseases. Sci Transl Med. 2021;13:eabe5640.

17. Byrnes KR, Wilson CM, Brabazon F, et al. FDG-PET imaging in mild traumatic brain injury: a critical review. Front Neuroenergetics. 2014;5:13.

18. Bajjalieh SM, Frantz GD, Weimann JM, McConnell SK, Scheller RH. Differential expression of synaptic vesicle protein 2 (SV2) isoforms. J Neurosci. 1994;14:5223-5235.

19. Lynch BA, Lambeng N, Nocka K, et al. The synaptic vesicle protein SV2A is the binding site for the antiepileptic drug levetiracetam. Proc Natl Acad Sci U S A. 2004;101:9861-9866.

20. Bertoglio D, Verhaeghe J, Miranda A, et al. Validation and noninvasive kinetic modeling of [(11)C]UCB-J PET imaging in mice. J Cereb Blood Flow Metab. 2020;40:1351-1362.

21. Finnema SJ, Nabulsi NB, Eid T, et al. Imaging synaptic density in the living human brain. Sci Transl Med. 2016;8:348ra396.

22. Cai Z, Li S, Matuskey D, Nabulsi N, Huang Y. PET imaging of synaptic density: A new tool for investigation of neuropsychiatric diseases. Neurosci Lett. 2019;691:44-50.

23. Bertoglio D, Verhaeghe J, Wyffels L, et al. Synaptic vesicle glycoprotein $2 \mathrm{~A}$ is affected in the CNS of Huntington's Disease mice and post-mortem human HD brain. $J$ Nucl Med. In press.

24. Toyonaga T, Smith LM, Finnema SJ, et al. In Vivo Synaptic Density Imaging with (11)CUCB-J Detects Treatment Effects of Saracatinib in a Mouse Model of Alzheimer Disease. J Nucl Med. 2019;60:1780-1786.

25. Lambeng N, Gillard M, Vertongen P, Fuks B, Chatelain P. Characterization of $[(3) \mathrm{H}] \mathrm{ucb}$ 30889 binding to synaptic vesicle protein 2A in the rat spinal cord. Eur J Pharmacol. 2005;520:7076.

26. Nicaise C, Putatunda R, Hala TJ, et al. Degeneration of phrenic motor neurons induces long-term diaphragm deficits following mid-cervical spinal contusion in mice. $J$ Neurotrauma. 2012;29:2748-2760.

27. Nicaise C, Hala TJ, Frank DM, et al. Phrenic motor neuron degeneration compromises phrenic axonal circuitry and diaphragm activity in a unilateral cervical contusion model of spinal cord injury. Exp Neurol. 2012;235:539-552.

28. Ross CA, Poirier MA. Protein aggregation and neurodegenerative disease. Nat Med. 2004;10 Suppl:S10-17. 
29. Bertoglio D, Deleye S, Miranda A, Stroobants S, Staelens S, Verhaeghe J. Estimation of the net influx rate $\mathrm{Ki}$ and the cerebral metabolic rate of glucose MRglc using a single static [(18)F]FDG PET scan in rats. Neuroimage. 2021;233:117961.

30. Deleye S, Verhaeghe J, wyffels L, Dedeurwaerdere S, Stroobants S, Staelens S. Towards a reproducible protocol for repetitive and semi-quantitative rat brain imaging with (18) F-FDG: exemplified in a memantine pharmacological challenge. Neuroimage. 2014;96:276-287.

31. Miranda A, Bertoglio D, Glorie D, Stroobants S, Staelens S, Verhaeghe J. Validation of a spatially variant resolution model for small animal brain PET studies. Biomed Phys Eng Expr. 2020;6.

32. Schiffer WK, Mirrione MM, Dewey SL. Optimizing experimental protocols for quantitative behavioral imaging with 18F-FDG in rodents. J Nucl Med. 2007;48:277-287.

33. Alizadeh A, Dyck SM, Karimi-Abdolrezaee S. Traumatic Spinal Cord Injury: An Overview of Pathophysiology, Models and Acute Injury Mechanisms. Front Neurol. 2019;10:282.

34. Oyinbo CA. Secondary injury mechanisms in traumatic spinal cord injury: a nugget of this multiply cascade. Acta Neurobiol Exp (Wars). 2011;71:281-299.

35. Assinck P, Duncan GJ, Hilton BJ, Plemel JR, Tetzlaff W. Cell transplantation therapy for spinal cord injury. Nat Neurosci. 2017;20:637-647.

36. Rossano S, Bini J, Nabulsi N, Ropchan J, Carson RE. Feasibility of imaging synaptic density in the human spinal cord using 11C-UCB-J PET. Paper presented at: Society of Nuclear Medicine and Molecular Imaging (SNMMI) 2021 Annual Meeting; May 2021, 2021.

37. Kalsi-Ryan S, Karadimas SK, Fehlings MG. Cervical spondylotic myelopathy: the clinical phenomenon and the current pathobiology of an increasingly prevalent and devastating disorder. Neuroscientist. 2013;19:409-421. 

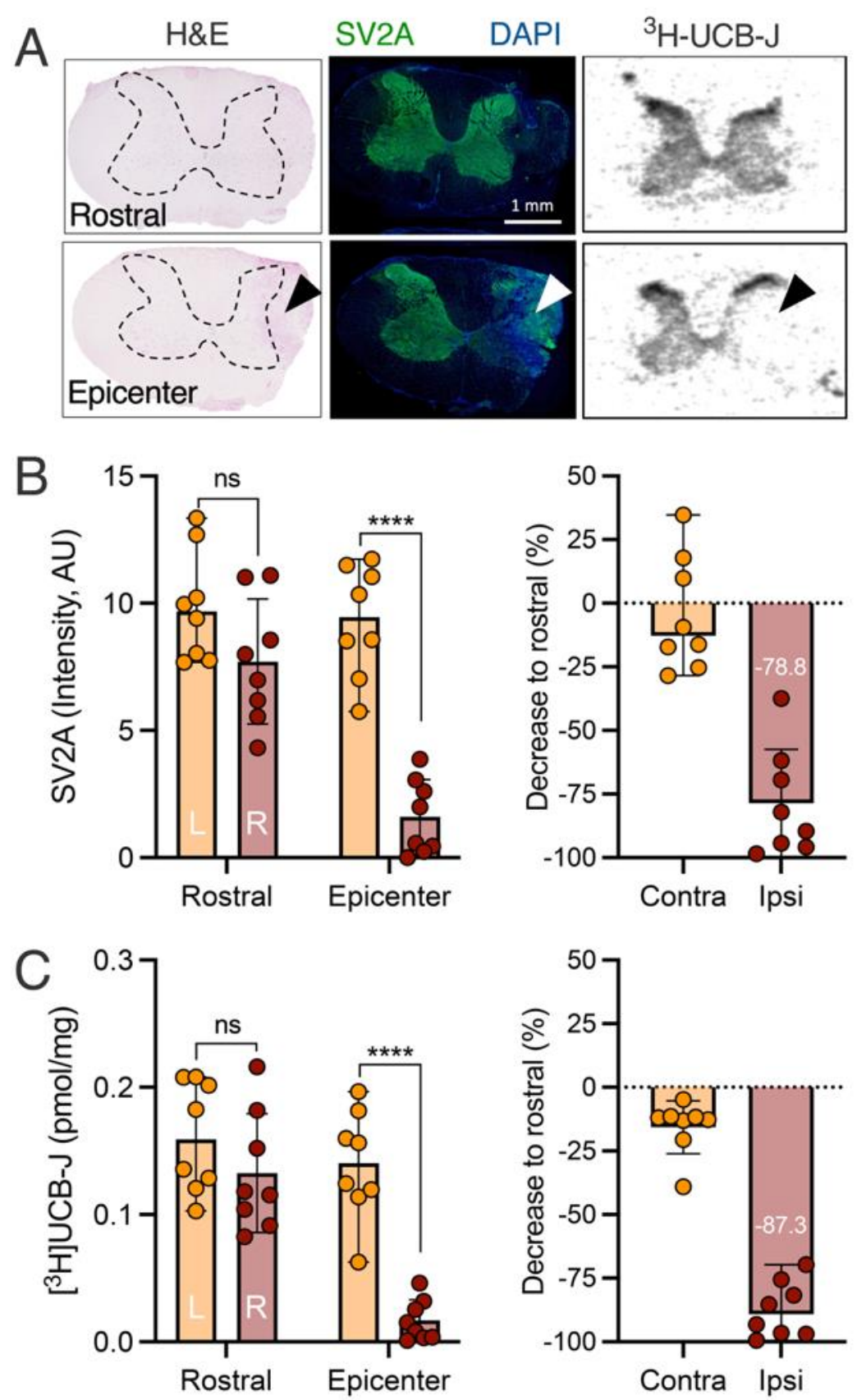

FIGURE 1. SV2A levels are decreased in the rat model of contusion SCI. (A) Representative H\&E, SV2A, and ${ }^{3} \mathrm{H}-\mathrm{UCB}-\mathrm{J}$ depicting rostral and epicenter spinal cord sections. Arrowheads indicate contusion site at right cervical C5, dashed lines outline spinal grey matter. SV2A (B) and ${ }^{3} \mathrm{H}-\mathrm{UCB}-$ $J(C)$ quantification in spinal cord sections and decrease in C5 section compared to rostral for both contralateral (Left) and ipsilateral (Right). ${ }^{* * * *} P<0.0001$. 


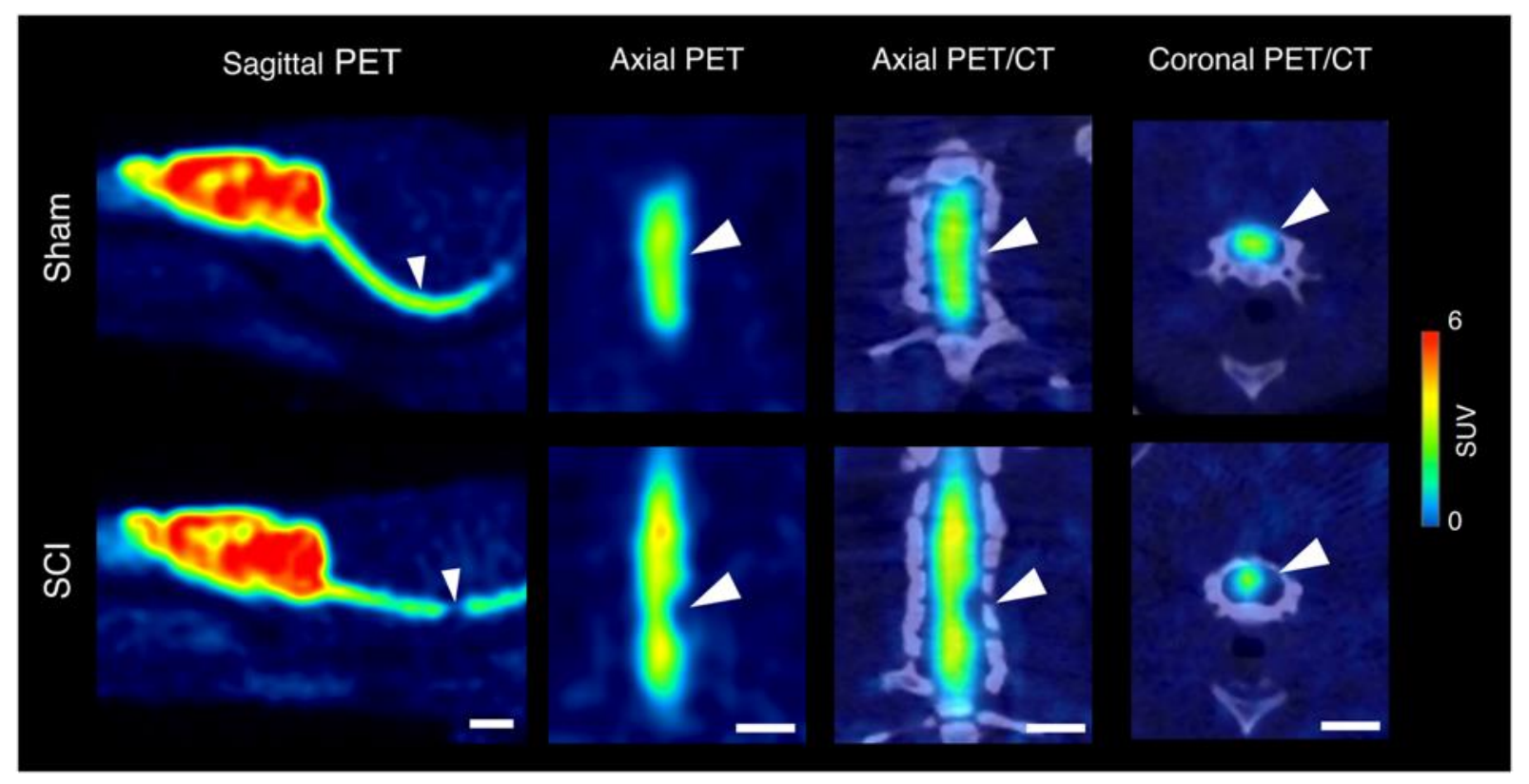

FIGURE 2. Representative ${ }^{11} \mathrm{C}-U C B-J$ PET image in a sham and a $\mathrm{SCI}$ rat 1 -week post-trauma. Arrowheads indicate a contusion site at cervical C5. Time interval 25-45 min post-injection. Scale bar $=5 \mathrm{~mm}$. 

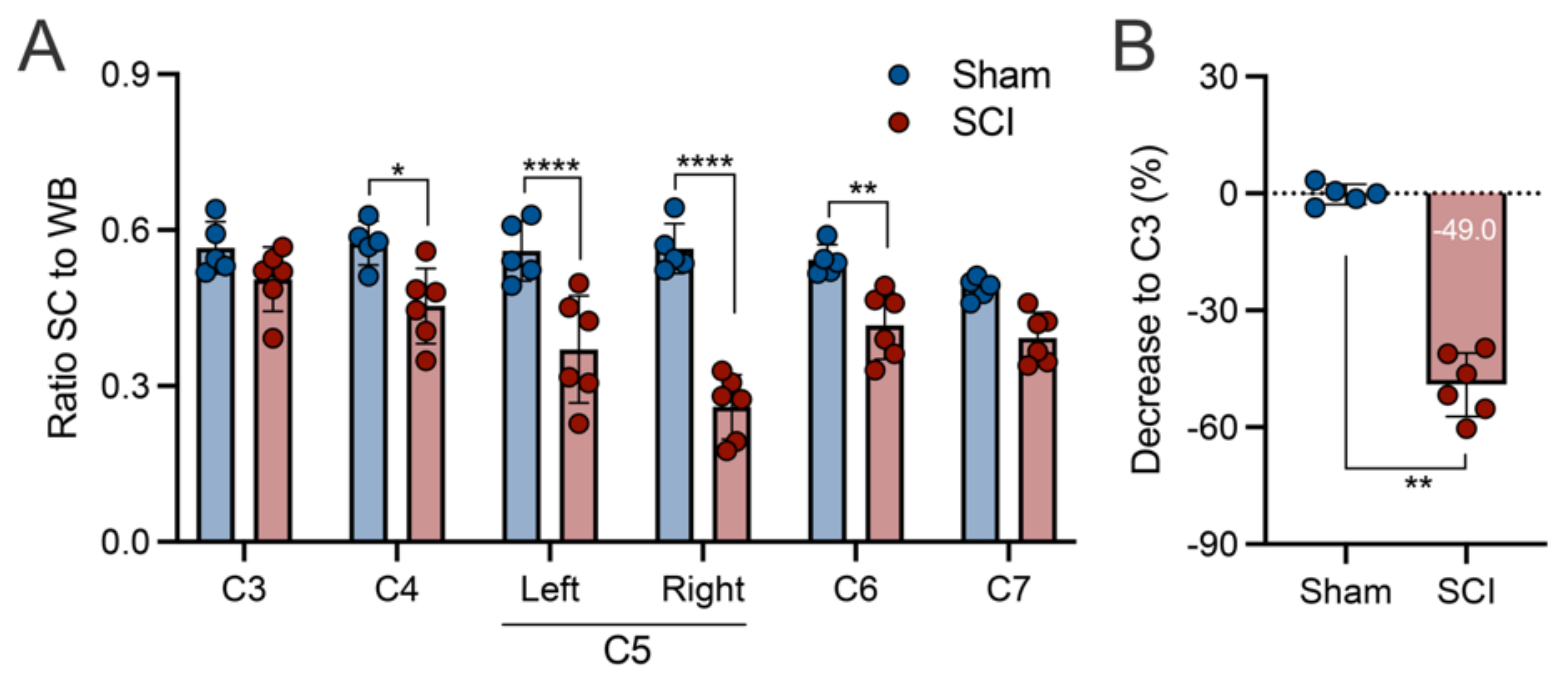

FIGURE 3. ${ }^{11} \mathrm{C}$-UCB-J PET detects SV2A loss in the contusion SCI rat model. (A) Spinal cord ${ }^{11} \mathrm{C}$ UCB-J uptake was significantly reduced around the contusion site (right C5), compared to sham animals. (B) Spinal cord ${ }^{11} \mathrm{C}-\mathrm{UCB}-\mathrm{J}$ uptake at epicenter (right C5) compared to C3 level within each animal. SC=spinal cord, WB=whole brain. ${ }^{*} P<0.05,{ }^{* *} P<0.01,{ }^{* * *} P<0.0001$. 

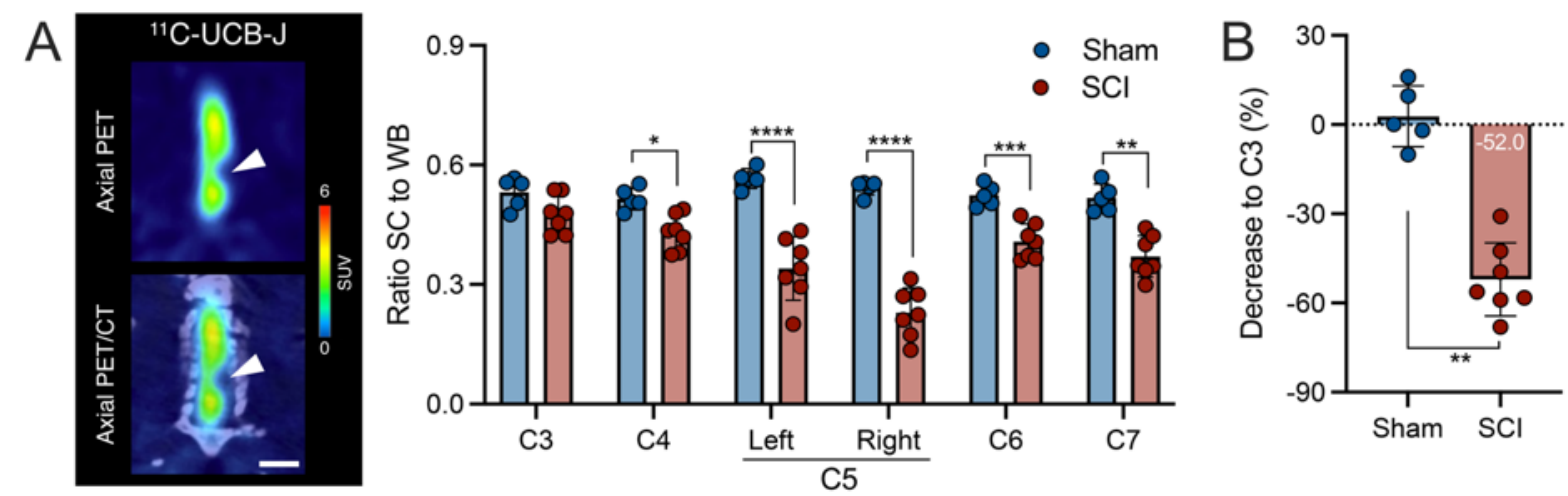

FIGURE 4. ${ }^{11} \mathrm{C}$-UCB-J reduction is sustained during chronic $\mathrm{SCl}$ in the contusion rat model. (A) Representative PET image in SCI rat and ${ }^{11} \mathrm{C}-\mathrm{UCB}-\mathrm{J}$ PET analysis in sham and rats with chronic SCI. Time interval 25-45 min post-injection. Scale bar=5 mm. (B) Spinal cord ${ }^{11} \mathrm{C}-\mathrm{UCB}-\mathrm{J}$ uptake at epicenter compared to $\mathrm{C} 3$ level within each animal. $\mathrm{SC}=$ spinal cord, WB=whole brain. ${ }^{\star} P<0.05$, ${ }^{* *} P<0.01,{ }^{* \star *} P<0.001,{ }^{* * *} P<0.0001$. 

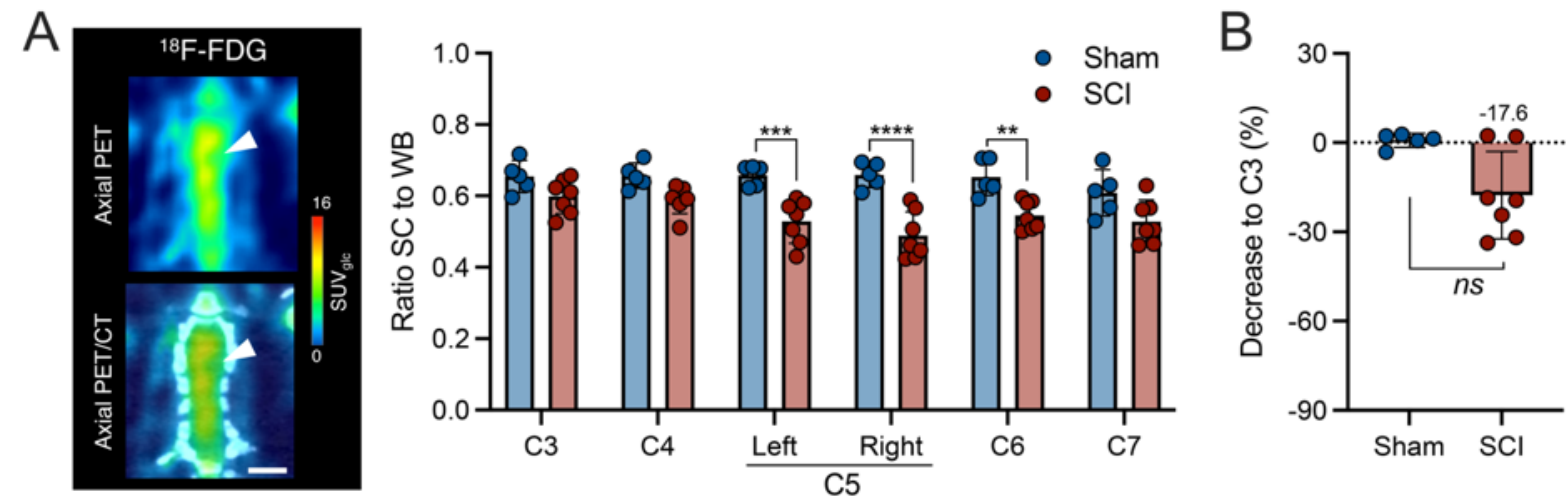

FIGURE 5. ${ }^{18} \mathrm{~F}-\mathrm{FDG}$ reduction is limited during chronic $\mathrm{SCl}$ in the contusion rat model. (A) Representative PET image in SCI rat and ${ }^{18} \mathrm{~F}$-FDG PET analysis in sham and with chronic SCl. Scale bar $=5 \mathrm{~mm}$. (B) Spinal cord ${ }^{18} \mathrm{~F}-\mathrm{FDG}$ uptake at epicenter compared to $\mathrm{C} 3$ level within each animal. SC=spinal cord, WB=whole brain. ${ }^{*} P<0.05,{ }^{* *} P<0.01,{ }^{* * *} P<0.001,{ }^{* * *} P<0.0001$. 

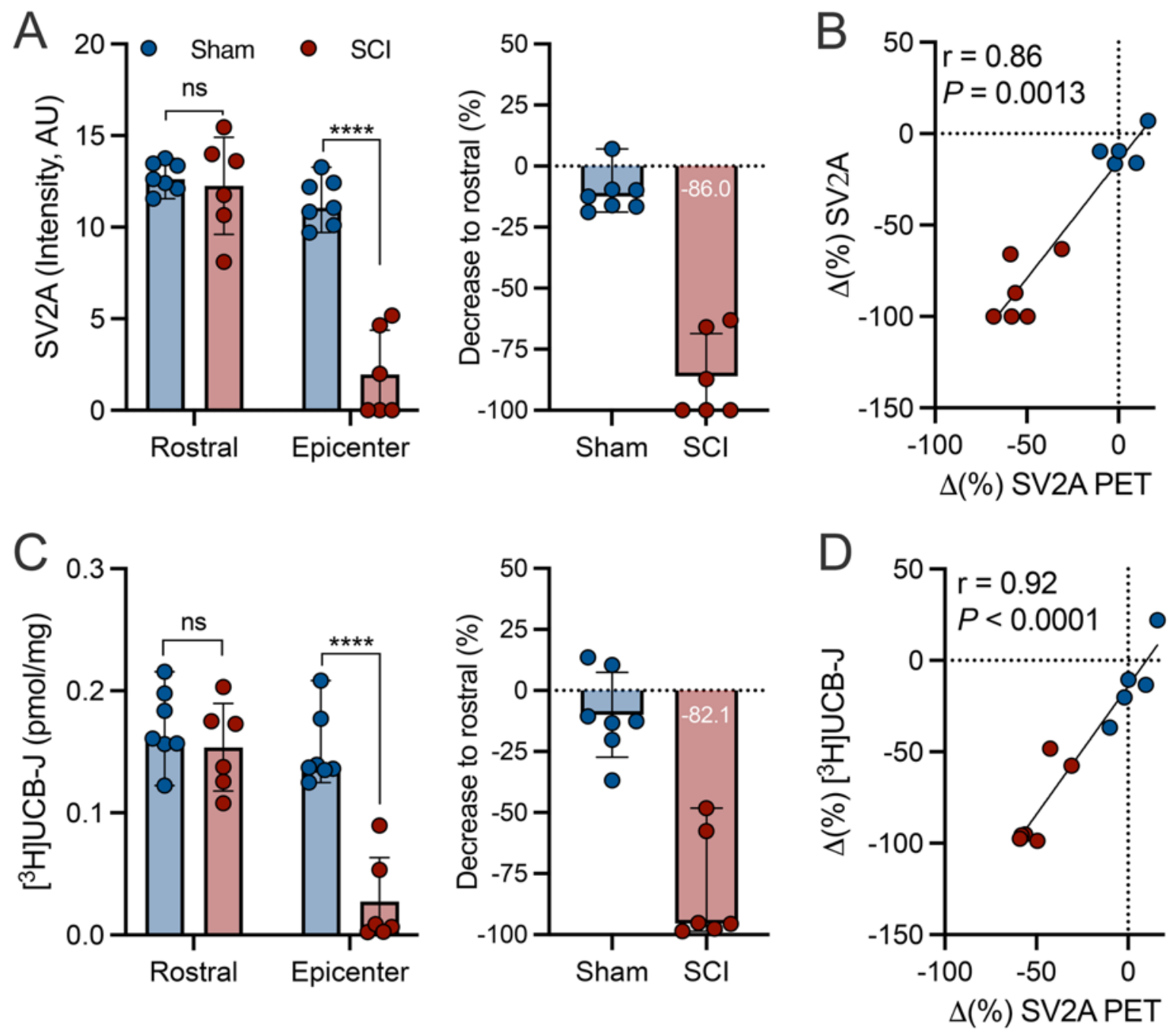

FIGURE 6. Post-mortem analyses corroborated the sustained SV2A loss measured with ${ }^{11} \mathrm{C}-\mathrm{UCB}-$ J PET during chronic SCI. (A) SV2A immunofluorescence quantification and (B) correlation between rostral to epicenter decrease measured with ${ }^{11} \mathrm{C}-U \mathrm{UCB}-J$ PET. (C) ${ }^{3} \mathrm{H}-U \mathrm{UCB}-\mathrm{J}$ autoradiography quantification and (D) correlation between rostral to epicenter decrease measured with ${ }^{11} \mathrm{C}-U C B-J$ PET. ${ }^{* * *} P<0.0001$. 


\section{SUPPLEMENTAL INFORMATION}

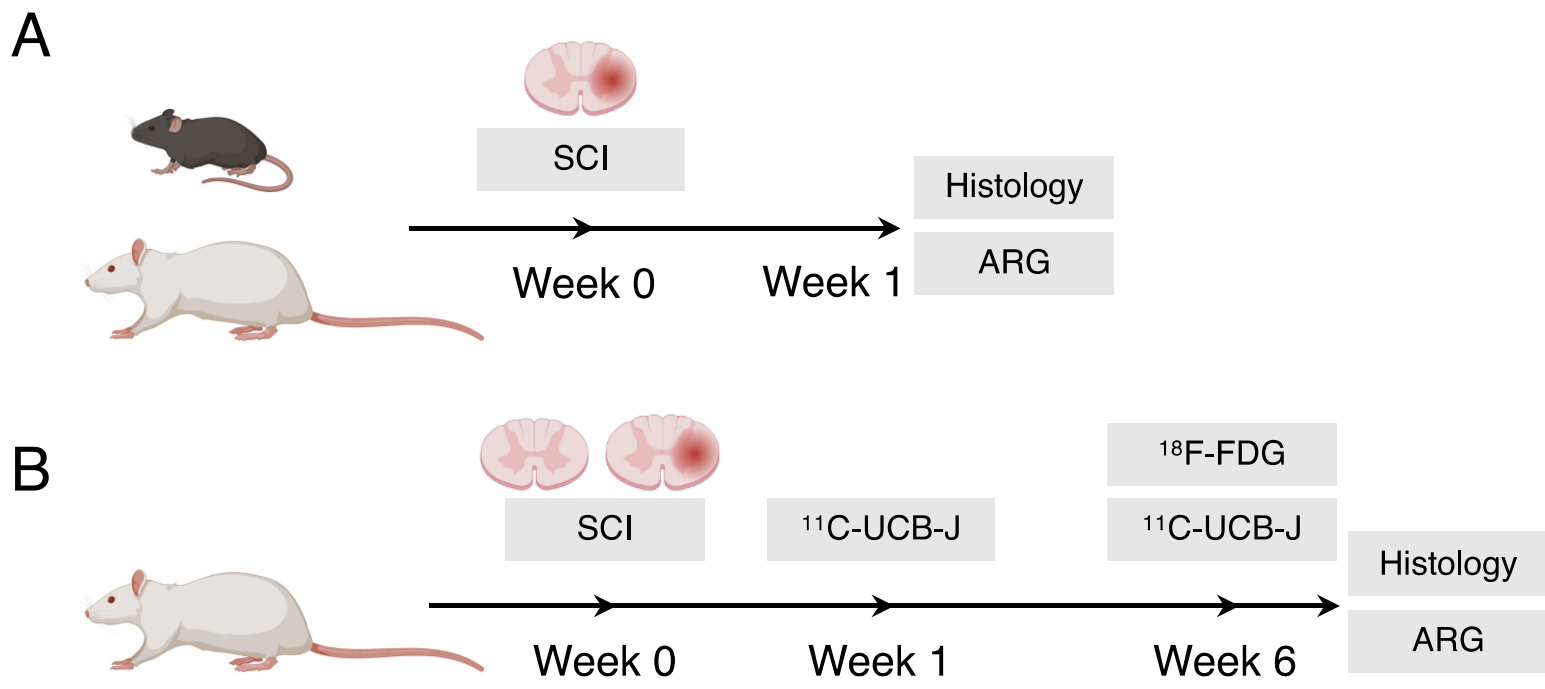

SUPPLEMENTAL FIGURE 1. Experimental outline describing the timeline of the spinal cord injury rodent models investigated and endpoints during the cross-sectional in vitro study (A) as well as the longitudinal in vivo study (B). SCl = spinal cord injury; $A R G=$ autoradiography. 


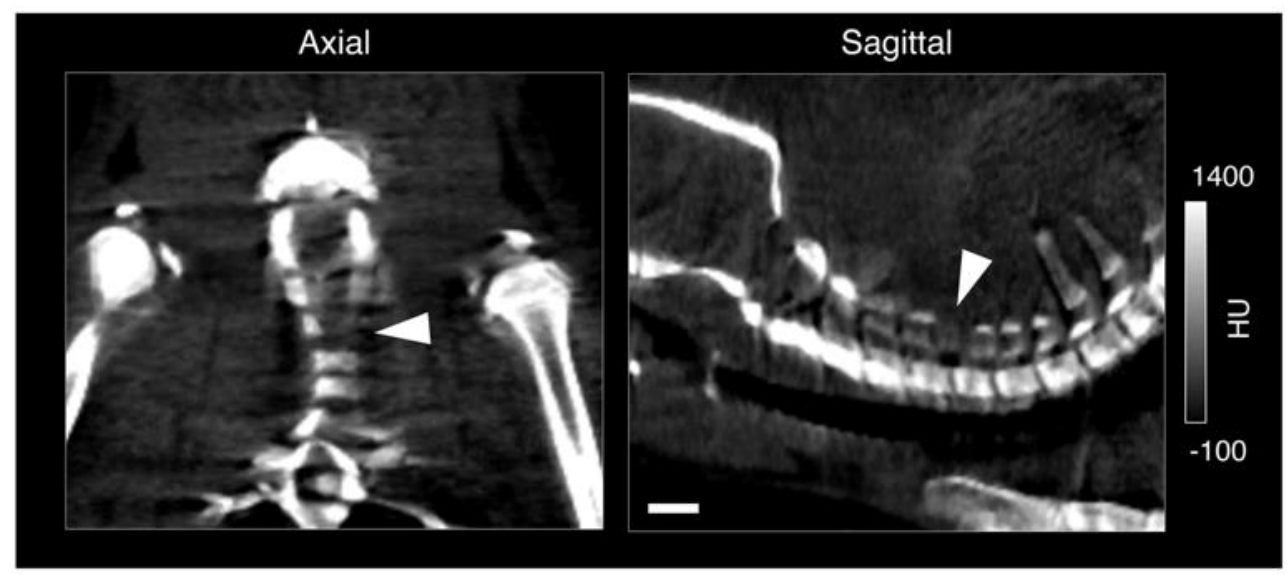

SUPPLEMENTAL FIGURE 2. Representative CT image in a sham rat 1-week post-surgery. White arrowhead indicates laminectomy at cervical C5. Scale bar $=5 \mathrm{~mm}$. 

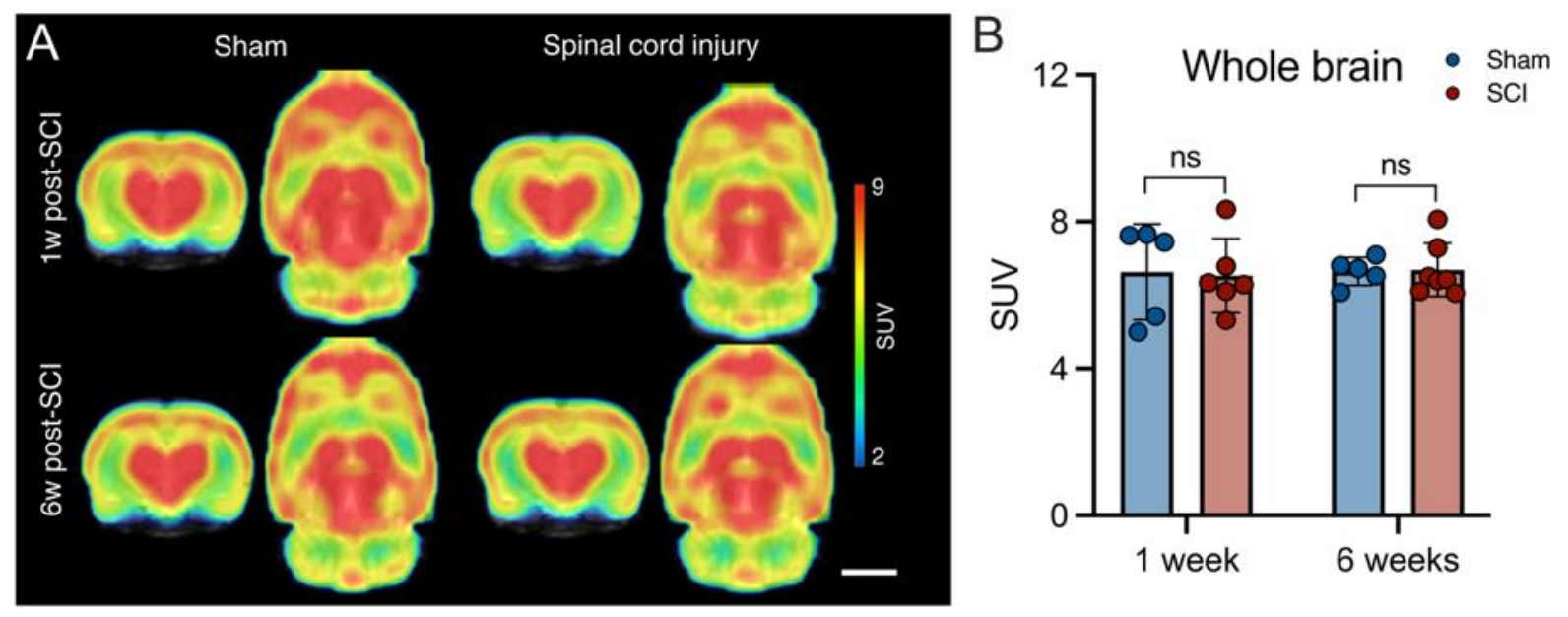

SUPPLEMENTAL FIGURE 3. Cerebral ${ }^{11} \mathrm{C}-U C B-J$ uptake. (A) Average cerebral standardized uptake value (SUV) images in sham and $\mathrm{SCl}$ rats at sub-acute and chronic SCI. Images are overlaid onto an MRI brain template for anatomical localization. Scale bar $=5 \mathrm{~mm}$. (B) Cerebral SUVs were unchanged between timepoints and groups. 

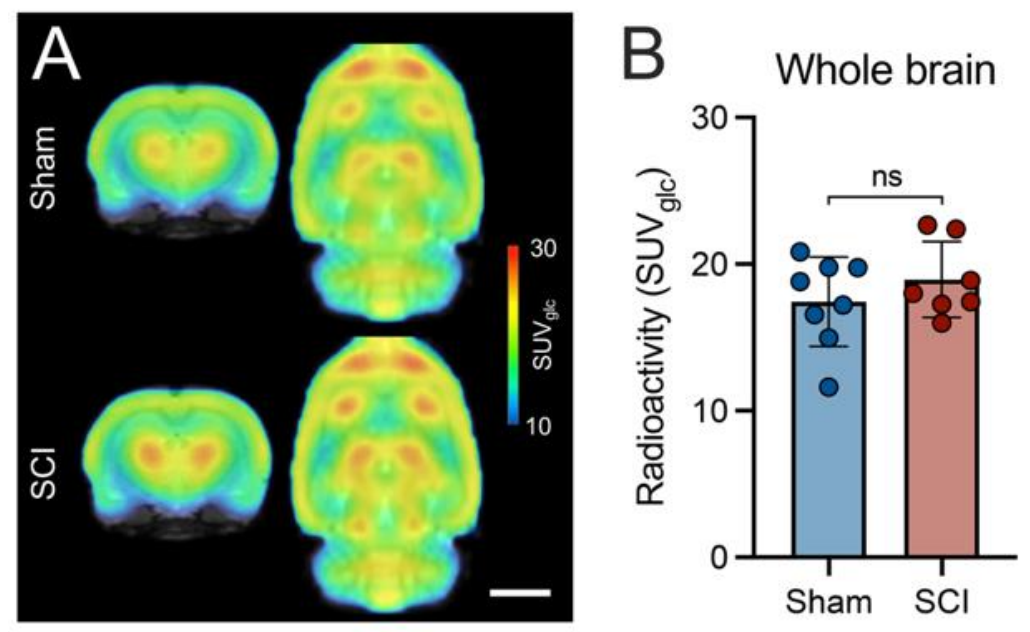

SUPPLEMENTAL FIGURE 4. Cerebral ${ }^{18}$ F-FDG uptake. Average cerebral glucose-corrected standardized uptake value $\left(\mathrm{SUV}_{\mathrm{glc}}\right)$ images $(\mathrm{A})$ and corresponding values $(\mathrm{B})$ were unchanged between sham and chronic SCI rats. PET images are overlaid onto an MRI brain template for anatomical localization. Scale bar $=5 \mathrm{~mm}$. 

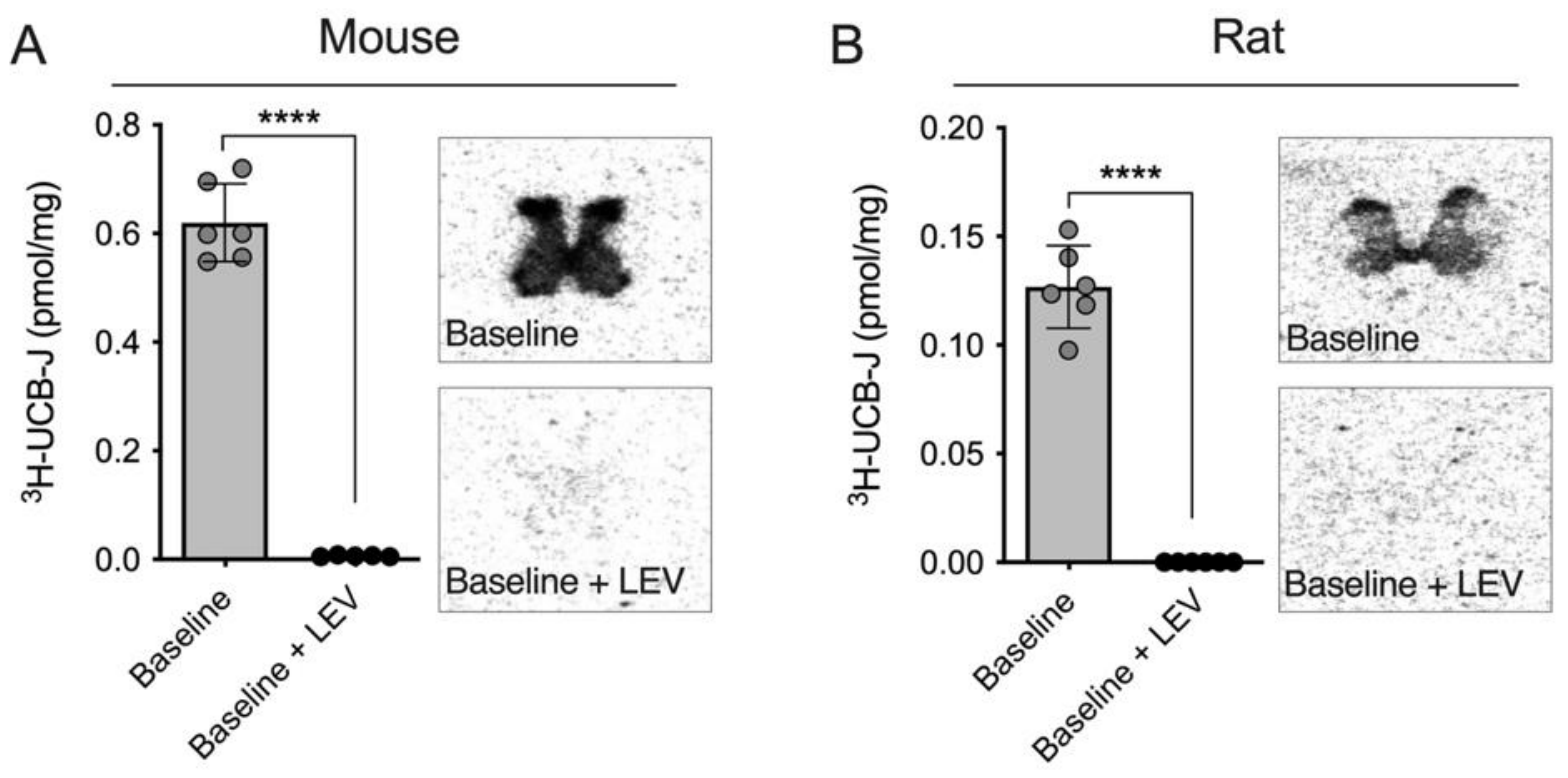

SUPPLEMENTAL FIGURE 5. ${ }^{3} \mathrm{H}$-UCB-J binding can be fully blocked in both the mouse (A) and rat (B) spinal cords. Blocking was obtained with levetiracetam (LEV, $1 \mathrm{mM}$ ) in adjacent slides of the same animal ( $n=6$ per species). ${ }^{* * *} P<0.0001$. 

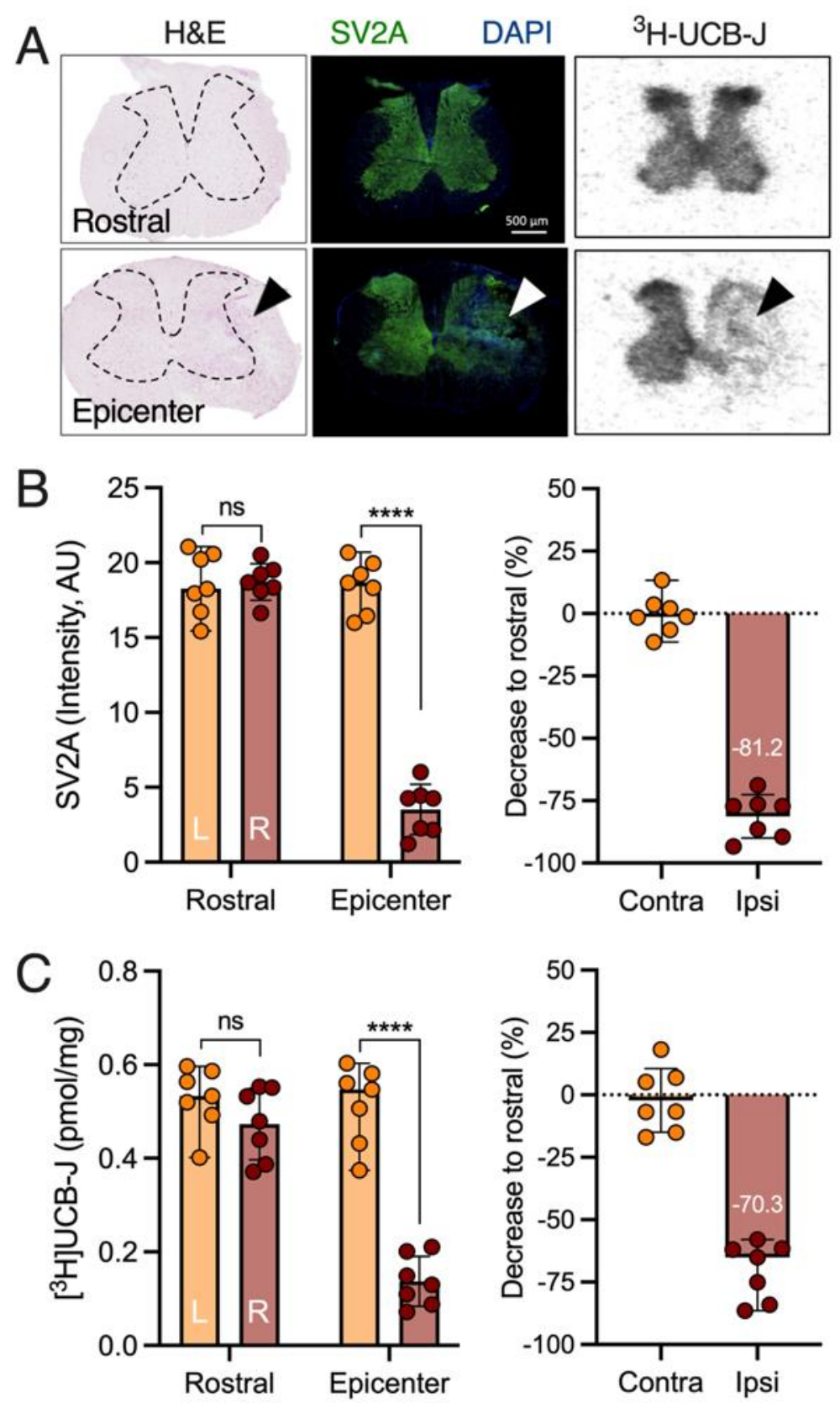

SUPPLEMENTAL FIGURE 6. SV2A levels are decreased in the mouse model of contusion SCI. (A) Representative H\&E, SV2A, and ${ }^{3} \mathrm{H}-\mathrm{UCB}-\mathrm{J}$ depicting rostral and epicenter spinal cord sections. Arrowheads indicate contusion site at right cervical C5, dashed lines outline spinal grey matter. SV2A (B) and ${ }^{3} \mathrm{H}-U C B-J(C)$ quantification in spinal cord sections and decrease in C5 section compared to rostral for both contralateral (Left) and ipsilateral (Right). ${ }^{* * *} P<0.0001$. 


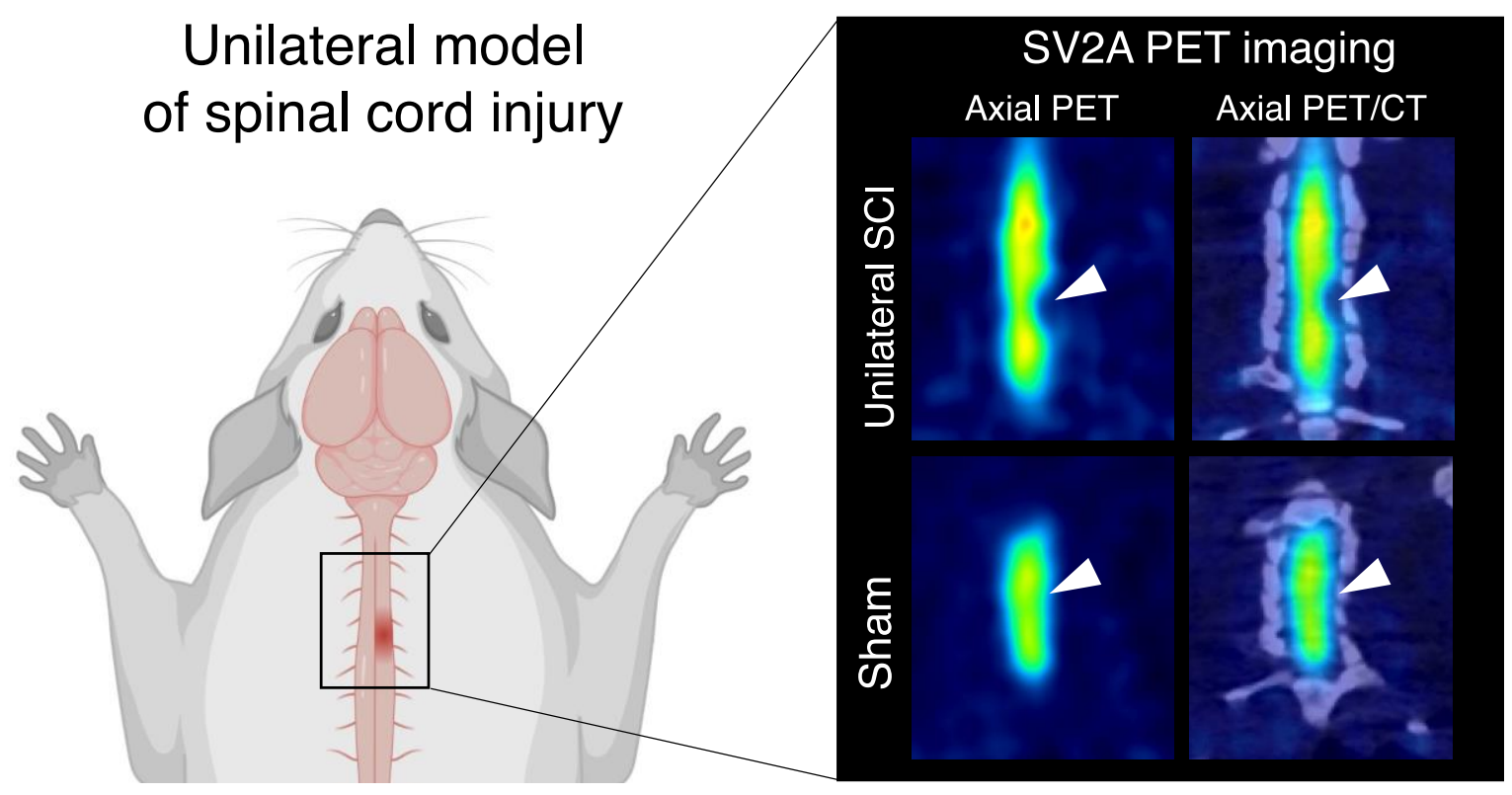

